\title{
21 Atomic Force Microscopy: Interaction Forces Measured in Phospholipid Monolayers, Bilayers, and Cell Membranes
}

\author{
Zoya Leonenko · David Cramb · Matthias Amrein · Eric Finot
}

\begin{abstract}
Atomic force microscopy (AFM) is a powerful technique which is commonly used to image surfaces at the nanoscale and single-molecule level, as well as to investigate physical properties of the sample surface using a technique known as force spectroscopy. In this chapter, we review our recent research where we used AFM to investigate physical properties of phospholipid monolayers, bilayers, and cell membranes. We describe the experimental procedures for AFM imaging, force measurements, and theoretical models to analyze force spectroscopy data. The data obtained allowed correlations between AFM topography and local adhesion and mechanoelastic properties of supported lipid bilayers in water, supported pulmonary surfactant films in air, and the plasma membrane of epithelial type II cells. Finally, AFM was applied to help elucidate the effect of anesthetics and cholesterol present in the lipid films.
\end{abstract}

Key words: Phospholipid, Monolayer, Bilayer, Pulmonary surfactant, Bovine lipid extract surfactant, Epithelial type II cell, Atomic force microscopy, Adhesion, Cholesterol, Halothane

\section{1 \\ Introduction}

Lipid membranes are integral components of the cellular organization in all living organisms [1]. As the major constituents of the biological membranes that form the outer boundary of cells and organelles they are involved in a vast number of biological processes. Their functions are to compartmentalize the cells, sort, regulate, and mediate biomolecular interactions. These complex functions are possible owing to the diverse physical-chemical properties of these membranes, which include two-dimensional fluidity, material elasticity, chemical diversity, and rich phase behavior [2]. Understanding the physical and chemical properties of lipid membranes $[3,4]$ and how they control biological processes is critical to our understanding of the molecular mechanisms of many diseases and finding a cure for them. The variety in structure and physical properties of cell membranes governs their biomolecular assembly and plays an important role in many fundamental biological processes [5]. For example, small, condensed rafts of spingolipids, cholesterol, and raft-associated protein play a crucial role in signaling, intracellular trafficking of lipids, platelet activation, membrane fusion, and protein binding [6,7]. Lipid rafts present in a cell membrane serve as entry and exit sites for microbial pathogens, such as influenza virus, measles virus, and HIV. Lipid-protein mixtures not only form membranes. Pulmonary surfactant $[8,9]$, which is a specific lipid-protein complex, 
forms a molecular film that covers the lung's interface with the air [10]. The major functions of pulmonary surfactant are to reduce the surface tension of the airspaceliquid interface, provide stability to the alveolar structure, and reduce the work of breathing [11]. Understanding the lateral distribution and interactions among the lipids and proteins in pulmonary surfactant monolayers is extremely important in order to develop methods of preventing alveolar collapse in cases of surfactant deficiency such as in premature infants or dysfunctional surfactant such as in adult respiratory distress syndrome (ARDS) [12].

Supported phospholipid bilayers (SPBs) [13] and monolayers are accepted and convenient model systems which mimic many fundamental properties of biological membranes and pulmonary surfactant. SPBs are composed of phospholipids adsorbed to a planar solid support. They also are of importance for biotechnology applications, as they form membrane-mimetic materials useful for creation of new classes of biosensors, diagnostic tools, and high-throughput characterization platforms for rapid and early detection of interactions between cells and their environment $[14,15]$. Such lipid films can serve as templates into which functional biomolecules can be incorporated, creating a powerful platform for the development of nanosensor and nanodevices. For biomedical and nanotechnology applications it is important to control processes involving the interaction of lipid membranes and monolayers with DNA and proteins, as well as polymers and inorganic nanoparticles. Adhesion of particles to the supported lipid films is important in biotechnology and medicine applications, for example, to study toxicity of nanoscale air pollutants and also for the development of effective pulmonary drug delivery systems [16].

Atomic force microscopy (AFM) has proven to be a valuable tool for imaging "soft" biological samples such as lipid films [17-21] especially in intermittent contact mode [22-24]. With AFM, one can not only image membranes in their native hydrated state. One can also measure the forces of interaction between the AFM probe and the sample, obtaining information about the physical properties of biological systems [25-29].

Measuring the physical and chemical properties of lipid membranes and monolayers involves measuring a multitude forces of weak noncovalent bonds (e.g., electrostatic, van der Waals, and/or hydrogen bonds) or hydrophobic interactions between geometrically complementary surfaces. To understand a response of a biological system to its environment one needs to measure the physical and chemical properties of the macromolecular assembly, such as membranes and monolayers, and how these properties are affected by mechanical stress, thermal change, and/or molecular adsorption. It concerns the mechanical properties such as two-dimensional fluidity and anisotropic elasticity of the membrane as well as thermodynamic properties underlying its rich phase behavior. Those bulk properties can also be investigated by a thermodynamic approach such as calorimetry, densitometry, contact angle measurement, or analysis of adsorption kinetics using surface plasmon resonance or ellipsometry. On a molecular level, molecular dynamics simulation has been employed, providing an insight into the organization of lipid structures and the stability. AFM is an ideal tool to measure directly the molecular force from $0.1 \mathrm{pN}$ up to micronewtons between two surfaces or even single molecules. On approach of the AFM probe tip to the sample, the force-distance curve can be used to characterize surface properties, such as van der Waals and electrostatic forces, solvation, 
hydration, and compression-related steric forces [30]. The retraction force curves often show a hysteresis referred to as an adhesion "pull-off" event, which can be used to estimate the adhesion forces. Many experimental force data are now available in the literature, and theoretical models have been developed for the analysis of forces acting between two solid surfaces [31-36]. In contrast, only a few publications have addressed force measurements of soft thin layers [37] such as thiol self-assembled monolayers [38-41] and phospholipid bilayers [42-44].

Here we review our recent research on interaction forces measured with AFM on SPBs in water and supported phospolipid monolayers in air, as models for biological membrane and pulmonary lung surfactant films, and on epithelial type II cells which cover the lung's interface with the air.

\section{2}

\section{Phase Transitions of Lipid Bilayers in Water}

The complex structural dynamics of phospholipid bilayers are governed by temperature-dependent parameters such as average interfacial area per lipid, bilayer thickness, and disorder of the hydrophobic tails. Although SPBs differ from free-standing membranes such as those found in vesicles or liposomes, they serve as good models for biological membranes. The SPB can exist in several lamellar phases: gel phase, liquid-crystalline or fluid phase, subgel phase, and ripple phase [45]. Often, phase behavior is dominated by the main $\mathrm{L}_{\beta}-\mathrm{L}_{\alpha}$ (gel-fluid) transition. A large number of stable, metastable, and transient lamellar gel structures can be adopted by different lipids. For saturated phosphatidylcholines, such as 1,2-dipalmitoyl phosphatidylcholine (DPPC), there are four recognized lamellar phases, namely, a liquidcrystalline phase $\left(\mathrm{L}_{\alpha}\right)$ or phases with ordered hydrocarbon chain arrangements, a ripple phase $\left(\mathrm{P}_{\beta}\right)$, a gel phase $\left(\mathrm{L}_{\beta}\right)$, and "subgel" or "crystal" phase $\left(\mathrm{L}_{\mathrm{c}}\right)$ [46]. Phase-related bilayer properties have been examined using a variety of experimental techniques [47-50]. The phase transition from the gel phase to the liquidcrystalline phase is shown schematically in Fig. 21.1. Physical properties of lipids such as density and thickness have been studied by differential scanning calorimetry, pressure calorimetry or dilatometry, and densitometry and other methods [51,52]. Temperature-dependent AFM studies allow for visualizing these phase transitions and provide valuable information about changes in structural and physical properties of bilayers during phase transitions. Temperature-dependent AFM studies involving imaging bilayers have been reported [53-57]. In addition, we have examined the force measurements as a function of temperature [58].

In this section we review our results on force measurements in SPBs during phase transitions associated with melting or anesthetic incorporation. Dioleoyl phosphatidylcholine (DOPC; lyophilized or in chloroform solution) (Sigma, Oakville, ON, USA) and DPPC (Avanti Polar Lipids, Alabaster, ME, USA) were used without further purification. Phosphate buffer $(\mathrm{pH}$ 6.8) and distilled, nanopure water were used in the preparation of all vesicles. Freshly cleaved ASTMV-2 quality, scratchfree ruby mica (Asheville-Schoonmaker Mica) was used as the solid support. Supported planar bilayers were prepared for AFM imaging by the method of vesicle fusion $[59,60]$. All vesicles were prepared as previously reported [59]. Aliquots of 
Fig. 21.1. Domain formation in a phospholipid bilayer supported on mica. The fluid phase domains correspond to a lower thickness than the gel-phase domains

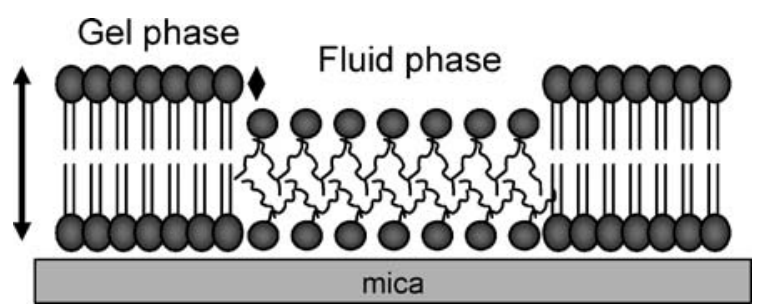

the vesicle solution were deposited on freshly cleaved pure mica. After a controlled period of time the mica was gently rinsed with nanopure water. Surfaces were imaged with an atomic force microscope (Pico SPM, Molecular Imaging) equipped with an AFMS-165 scanner. The nominal spring constants of Au-Cr-coated MAClevers used were approximately $0.6 \mathrm{~N} / \mathrm{m}$. The tip radius of curvature was typically $25 \mathrm{~nm}$. The scan rate was $20 \mu \mathrm{m} / \mathrm{s}$. All imaging and force measurements were performed in a liquid cell under nanopure water. For temperature-dependence experiments the temperature was varied from 22 to $65^{\circ} \mathrm{C}$, within $0.1^{\circ} \mathrm{C}$ accuracy with a $1{ }^{\circ} \mathrm{C} / \mathrm{min}$ ramp.

\subsection{1}

\section{Morphology Change During Lamellar Phase Transition}

\subsubsection{1}

\section{Temperature-Dependent Phase Transition}

A DPPC phospholipid bilayer normally exists in the gel phase at room temperature. When heated above the melting transition temperature it transforms into the fluid phase. Domains of lower thickness are readily formed during this process. Such domains are shown schematically in Fig. 21.1 and are easily observed by AFM imaging (Fig. 21.2).

A DPPC supported bilayer was imaged while it was heated from room temperature to $65^{\circ} \mathrm{C}$ and also while it was cooled back to room temperature. The topography images (Fig. 21.2) show the typical formation of lipid domains during the phase transition. In Fig. 21.2, panel A, a DPPC bilayer is in the gel phase, $\mathrm{L}_{\beta}$, at $22^{\circ} \mathrm{C}$. When sample was heated, several phase transitions were observed as a function of increasing temperature: a broad main transition was observed at $42-52^{\circ} \mathrm{C}$, and another transition at $53-60^{\circ} \mathrm{C}$ within the fluid phase. The first transition, at $42-52{ }^{\circ} \mathrm{C}$, is attributed to the main $\mathrm{L}_{\beta}-\mathrm{L}_{\alpha}$ transition, and the second transition, between $53-60^{\circ} \mathrm{C}$, to the formation of a fluid disordered phase, perhaps with interdigitated or partly interdigitated lipid chains. Slowly cooling the system back showed that the two higher-temperature transitions at $42-52$ and $53-60{ }^{\circ} \mathrm{C}$ are reversible and that the bilayer can be restored to its initial thickness [61]. A DOPC bilayer, which exists in a fluid phase at room temperature, when heated shows thickness reduction as well as an increase in surface coverage [62], but no domain formation. Previous studies have established the $\mathrm{L}_{\beta}-\mathrm{L}_{\alpha}$ phase transition temperature for DPPC at $41^{\circ} \mathrm{C}$ [63]. The presence of two broad transitions that we observed on supported bilayers can be attributed to the effect of the mica support and to separating the phase transitions 


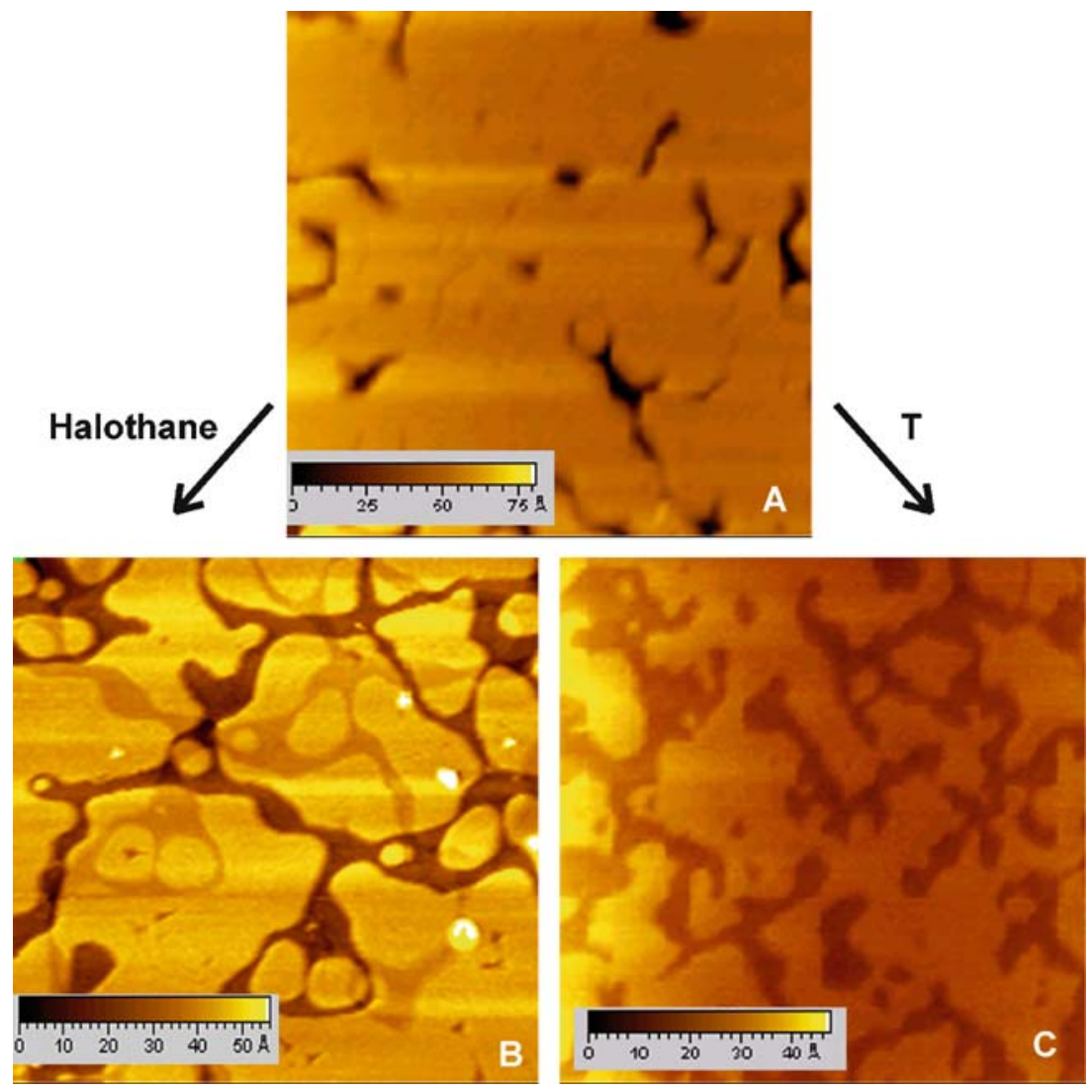

Fig. 21.2. Atomic force microscopy (AFM) topography images (scan size $1.5 \mu \mathrm{m}$ by $1.5 \mu \mathrm{m}$ ) showing a phase transition in a 1,2-dipalmitoyl phosphatidylcholine (DPPC) bilayer. A DPPC bilayer at room temperature, $22^{\circ} \mathrm{C}$. B DPPC bilayer with excess of halothane $(60 \mu \mathrm{l})$ [57]. C $50{ }^{\circ} \mathrm{C}$-melting transition [61]. The scale bars show the deviation in height

in the bilayer's upper and lower leaflets. A similar effect was reported for supported bilayer transitions observed using differential scanning calorimetry by Yang and Appleyard [64].

\subsubsection{2}

\section{Phase Transition Due to Incorporation of Halothane}

Understanding the changes in physical and chemical properties of biological membranes owing to the incorporation of anesthetics is of great interest for understanding the mechanism of anesthetic action. Currently, it is understood that anesthetic directly affects membrane proteins; however, because they are lipophilic, they partition into the membrane and may interact with membrane proteins via the lipid bilayer.

AFM imaging of a DPPC bilayer (Fig. 21.2, panel B) shows domain formation in the presence of general anesthetic halothane similar to what we observed during 
heating. We have shown that changes in bilayer thickness induced by halothane are similar to the first melting transition [58]. For a DPPC SPB containing halothane, only one transition at $52-60^{\circ} \mathrm{C}$ was evident during heating and cooling. The presence of halothane changes the structural properties of the bilayer in such a way that the other transitions observed for the pure bilayer are not possible. We assume that in the presence of halothane, the water interface layer is disturbed by halothane molecules, thus eliminating the low-temperature transition. In spite of the visual similarity between an anesthetic-induced domain formation (Fig. 21.2, panel B) and the heat-induced gel-liquid phase transition (Fig. 21.2, panel C), observed by AFM imaging, the mechanism of anesthetic action is likely to be different from the effect of membrane melting. We assume that the physical properties of the domains produced in lipid bilayers by halothane and by temperature are also different. To address these physical properties we used the atomic force microscope also as a force apparatus.

\subsection{2}

\section{Change in Forces During Phase Transition}

\subsubsection{1}

\section{Force Measurements}

In AFM force measurements, the probe is moved towards the sample and cantilever deflection is measured as a function of the extension of the piezoelectric tube. During approach to the surface, the attractive and repulsive forces measured are characterized by van der Waals and electrostatic interactions as well as solvation, hydration, and compression-related steric forces $[26,36,52,65]$. The probe is then retracted from the surface. The retraction force curves often show a hysteresis referred to as an adhesion pull-off event, which can be used to determine adhesion forces between the probe and the sample.

In our experiments, we imaged the supported bilayer first in tapping MAC mode. After the image was complete and had been saved we switched the regime into the contact mode and then using the same cantilever, we measured forces of interaction by positioning the atomic force microscope tip on different locations using the image obtained in tapping mode.

It is a well-known fact that the force interactions depend on the velocity of the surface approach [26], especially for soft, viscoelastic materials. We varied the frequency sweep over about four decades, from 0.01 to $10 \mathrm{~Hz}$. The $Z$-range was $50 \mathrm{~nm}$. Note that varying the scan frequency affects simultaneously the velocity at which the tip is withdrawn from the bilayer and the contact time between the tip and the sample. At a fixed applied load, increasing the frequency sweep rate increases the tip velocity, but decreases the contact time. Data were collected over a time period of $2 \mathrm{~h}$ at ten locations on the bilayer and a total of 100 force curves were analyzed for each sample. Force curves were saved as plots and then converted into text files for analysis. After the raw data had been collected they were converted into the "force versus separation plots", averaged, and analyzed.

Here we review our results on force measurements in DOPC and DPPC SPBs during heating above $T_{\mathrm{M}}$ from 22 to $65^{\circ} \mathrm{C}$. We also compare them with results obtained on bilayers saturated with halothane at $20^{\circ} \mathrm{C}$. 


\subsubsection{2}

\section{AFM Force Analysis}

Force measurements using the atomic force microscope (described in Sect. 21.2.2.1) give us raw data of cantilever deflection $\delta V$ versus piezo movement $z_{\text {piezo }}$. Raw data were transformed numerically using the MatLab environment. Raw data $\left(Z_{\mathrm{c}}\right.$ versus $Z_{\mathrm{p}}$ ) were then converted into force $F$ versus surface-tip separation $D$ using Hooke's law: $F=k Z_{\mathrm{c}}$, where $k$ is the spring constant of the cantilever, and the geometric relationship $D=Z_{\mathrm{c}}-Z_{\mathrm{p}}$ for incremental changes.

Statistical data were extracted from a large set of measurements. The bin size of the histogram was determined statistically by dividing the range of measurements by the square root of the number of measurements. The average of the adhesion force and the standard deviation were obtained from the analysis of the whole set of force curves, which were represented in histogram form, $P(x)$, and then adjusted using Gaussian and Poisson distributions.

\subsubsection{3}

\section{Adhesion Forces}

To extract information about adhesion forces we analyzed the retrace part of the force curve. Raw data of the cantilever deflection corresponding to the retraction of the tip from the surface, after contact, are shown in Fig. 21.3.

Force data were collected as a function of temperature for a DPPC SPB. When a pure DPPC bilayer was heated, the temperature increase resulted in a gradual change of the force profile. The force required to detach the tip from the bilayer increased from $3 \mathrm{nN}$ at $36^{\circ} \mathrm{C}$ to $10 \mathrm{nN}$ at $50^{\circ} \mathrm{C}$. In addition, the jump out of the contact was not abrupt, compared with that for a "solid-like" surface (i.e., mica). The distance the tip travels before it separates from the surface increased with increasing temperature. As van der Waals forces are known to vary only slightly with temperature, the change in the profile must be due to tip-bilayer interactions that depend on the bilayer phase. Incorporation of halothane into DPPC also leads

Fig. 21.3. Cantilever deflection $d Z_{c}$ as a function of the piezotube elongation when the tip is retracted from the sample. DPPC bilayers deposited on mica were measured under three conditions: a the DPPC bilayer at $22^{\circ} \mathrm{C}$; b the DPPC bilayer at $50^{\circ} \mathrm{C}$; $\mathbf{c}$ the DPPC bilayer with halothane incorporated at $22{ }^{\circ} \mathrm{C}$

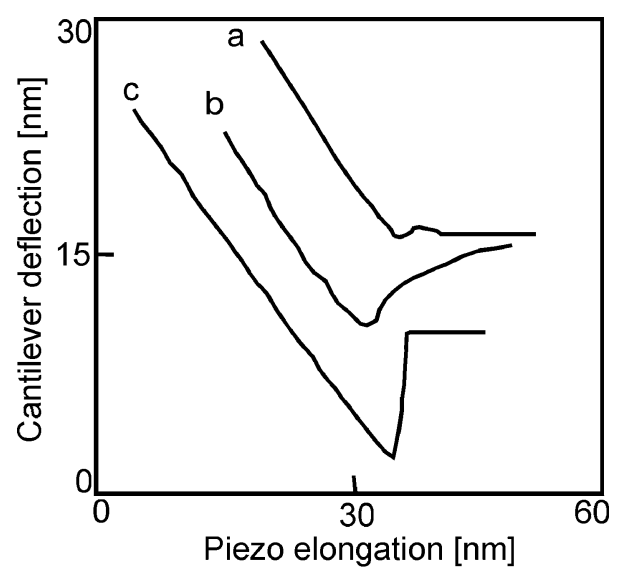


to a significant change in the force profile. As a bilayer adsorbed on mica is known to screen the van der Waals forces [66], the huge adhesion force observed cannot originate solely from the van der Waals forces. This adhesion behavior can be compared with that observed in the phase transition on DPPC from the gel to the liquid crystal observed on increasing the temperature (Fig. 21.3, plots b and c). Halothane induces then a transition of the bilayer into the fluid phase in a similar manner as melting, and induces the increase in adhesion, although the adhesion with excess halothane was greater than the adhesion observed on pure DPPC at elevated temperature.

To analyze the force histogram (Fig. 21.4), we employed Poisson statistics. It has been demonstrated that the force of a unit interaction between an atomic force microscope tip and a surface can be determined from a statistical analysis of a series of detachment force measurements. For a statistical analysis based on adhesion force originating from a discrete number $n$ of individual interactions or bonds, $F_{\mathrm{s}}$ was used. The total force distribution follows Poisson statistics, where both the adhesion force $F_{\text {adh }}$ and the variance $\sigma$ originate from a number of individual tip-surface bonds, $n$ :

$$
F_{\text {adh }}=n F_{\mathrm{s}} \quad \text { and } \quad \sigma^{2}=n F_{\mathrm{s}}^{2} .
$$

The force of one bond, $F_{\mathrm{s}}$, is therefore given by the square of the variance of the force divided by the mean adhesion force $F_{\text {adh }}$. The value $n$ is the ratio between $F_{\text {adh }}$ and $F_{\mathrm{s}}$. This analysis has the advantage that knowledge of the mean radius of curvature is not required [67] and gives information on the nature of the bilayer. This analysis allows one to correlate microscale changes in the bilayer physical properties with
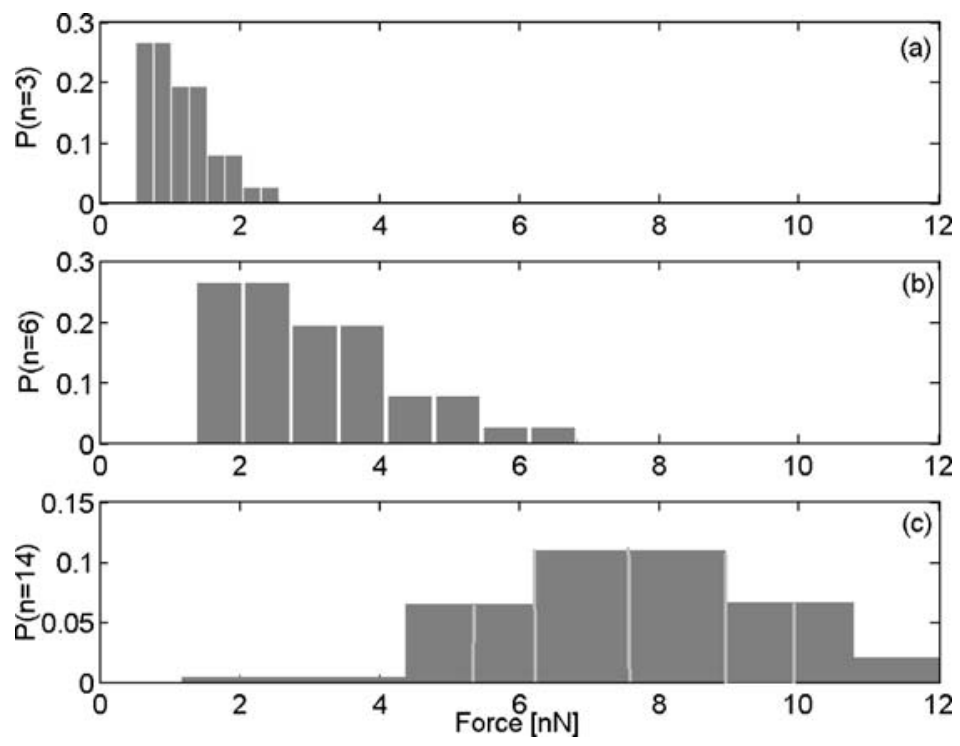

Fig.21.4. Distribution of adhesion force $F_{\text {adh }}$, measured in phospholipid bilayers in water: a DPPC $F_{\mathrm{s}}=0.3 \mathrm{nN}, n=3 ;$ b DPPC $F_{\mathrm{s}}=0.5 \mathrm{nN}, n=6$ [58]; $\mathbf{c}$ DPPC + halothane $F_{\mathrm{s}}=0.5 \mathrm{nN}$, $n=14[58]$ 
Table 21.1. Experimental adhesion force $F_{\text {adh }}$ and its standard error $\sigma$ on a 1,2-dipalmitoyl phosphatidylcholine (DPPC) bilayer at room and elevated temperature and DPPC with halothane, measured at room temperature; calculation of the number of bonds $n$ and the mean force of a single bond $F_{\mathrm{s}}$

\begin{tabular}{llllll}
\hline & $\begin{array}{l}\mathrm{DPPC}^{\mathrm{a}} \\
22{ }^{\circ} \mathrm{C}\end{array}$ & $\begin{array}{l}\mathrm{DPPC}^{\mathrm{b}} \\
2{ }^{\circ} \mathrm{C}\end{array}$ & $\begin{array}{l}\mathrm{DOPC}^{\mathrm{a}} \\
2{ }^{\circ} \mathrm{C}\end{array}$ & $\begin{array}{l}\mathrm{DPPC}^{\mathrm{a}} \\
60{ }^{\circ} \mathrm{C}\end{array}$ & $\begin{array}{l}\mathrm{DPPC}+\text { halothane }^{\mathrm{b}} \\
22^{\circ} \mathrm{C}\end{array}$ \\
\hline$F_{\mathrm{adh}}(\mathrm{nN})$ & 1 & 1.5 & 3 & 5 & 7 \\
$\sigma(\mathrm{nN})$ & 0.6 & 0.8 & 1.2 & 2 & 2 \\
$F_{\mathrm{S}}(\mathrm{nN})$ & 0.3 & 0.4 & 0.5 & 0.8 & 0.5 \\
$n$ & 3 & 4 & 6 & 6 & 14 \\
\hline
\end{tabular}

DOPC dioleoyl phosphatidylcholine

${ }^{\text {a }}$ From [61]

${ }^{\mathrm{b}}$ From [58]

the molecular structure and the mobility of individual lipid molecules. The lipids in biological membranes have a high lateral mobility since they can easily exchange their positions. The fluidity of the membrane can be then thought of as the lateral motion of the constituents of the membranes. This fluidity should depend on the chain length and the composition of the membranes. The mobility of tails can be seen as the number of molecules $n$ in contact with the AFM probe. The larger $n$ is, the higher the mobility. The change in mobility increases the number of bonds $n$ and therefore $F_{\text {adh }}$ and $\sigma$.

The statistical distribution of the adhesion forces (Table 21.1) clearly demonstrates that adhesion forces observed for a pure DPPC bilayer increase with heating and increase even more with halothane incorporation.

A fluid-phase DPPC bilayer at high temperature is typically ascribed to a higher number of tip-sample bonds [61]. The force of a single bond is increased in a fluid phase. In addition, in a fluid phase, $n$ increases on decreasing the speed of the tip motion $v$. The number of bonds, $n$, involved is higher in the fluid phase. It has been shown previously that the force required to remove a single lipid from a bilayer is of the order 30-140 $\mathrm{pN}[68,69]$, depending on the lipid and on the technique employed. The lowest values that we measure are at least twice this range, suggesting that we do not observe single lipid binding events. Instead, our unit "bonds" must represent patches containing more than three lipids. The detachment force between the patch and the tip must be smaller than the extraction force of the entire patch otherwise we would see evidence of lipids coating the tip. This is consistent with our previous work where continuous imaging of bilayers displayed no resolution degradation or changes in force curves, which would result from uncontrollable lipid coating the atomic force microscope tip.

The effect of halothane is similar to the effect of melting. A DPPC bilayer with halothane corresponds to higher adhesion force, higher standard error, and a larger number of single binding events between the atomic force microscope tip and the sample. This number, $n=14$, is more than 2 times larger than for a fluid phase produced by melting. The single force $F_{\mathrm{s}}$ was not altered significantly by the presence of halothane. The presence of halothane in the bilayer increases, 
therefore, the fluidity of the DPPC molecules in the bilayer. The number of binding events, $n$, could also be higher owing to the contribution of halothane molecules themselves, interacting with the atomic force microscope tip. The unit force $F_{\mathrm{s}}$ for fluid-phase DPPC is 0.8 compared with 0.5 for the DOPC bilayer (Table 21.1); the umber of unit bonds is 6 for both fluid-phase DPPC and DOPC bilayers. This correlation permits evaluation of a bilayer fluidity and is in a good agreement with imaging data. Adhesion forces depend, therefore, on the physical properties of the bilayer and the force analysis gives valuable information on the nature of the bilayer.

\subsubsection{4}

\section{Repulsive Forces}

Repulsive forces were extracted by analyzing the approach part of experimental force curves. Figure 21.5 shows plots of the repulsive forces measured while the atomic force microscope tip was approaching the sample surface of pure mica for a DPPC bilayer at room temperature, a DPPC bilayer at $50^{\circ} \mathrm{C}$ [61], and a DPPC bilayer with an excess of halothane [58].

For pure DPPC, three exponential regimes clearly exist, one at the long range from 4 to $7 \mathrm{~nm}$, another at short range between 0.3 and $4 \mathrm{~nm}$, and the third, steep one in close contact with mica, which we will not consider.

The long-range interactions can be explained by electrostatics. Electrostatic repulsion likely originates from an effective charge density at the bilayer-water interface, where zwitterionic headgroups interact with water molecules. The approximate expression of the electrostatic force (also called double-layer force) is given by [70]

$$
F=4 \pi \sigma R \psi_{\mathrm{T}} \exp \left(-K_{\mathrm{D}} D\right)
$$

where $R$ is the tip radius, $\sigma$ is the surface charge, $\psi_{\mathrm{T}}$ is the tip potential, and $K_{\mathrm{D}}$ is the inverse of the Debye length. $K_{\mathrm{D}}$ can be estimated to be $0.25 / \mathrm{nm}$.

Fig. 21.5. Logarithmic representation of the force acting on the DPPC layer at $22^{\circ} \mathrm{C}$ (squares), on the DPPC layer at $50{ }^{\circ} \mathrm{C}$ (circles), and on the DPPC layer with halothane at $22^{\circ} \mathrm{C}$ (triangles)

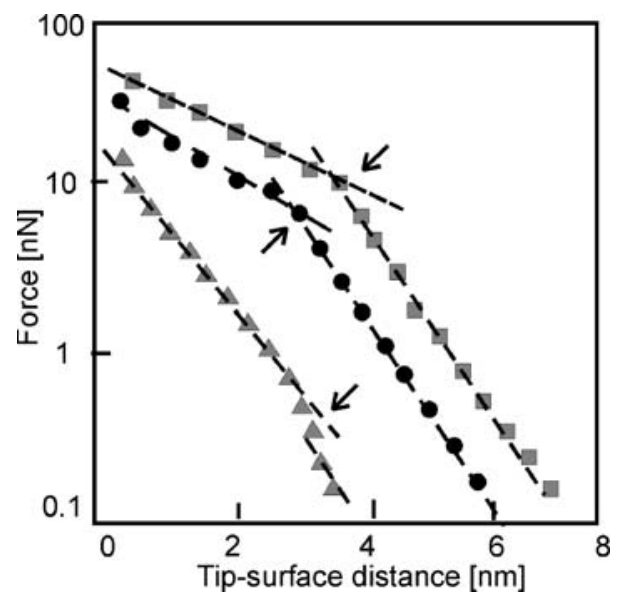


The long-range force does not seem to be affected by temperature, as the two curves in the log plot are almost parallel (Fig. 21.5). In the presence of halothane, (Fig. 21.5), the long-range electrostatic forces appear to be reduced, in the range above $4 \mathrm{~nm}$. Therefore, halothane clearly changes the surface charge density of the DPPC bilayer by replacing water molecules and reducing water shielding of the lipid bilayer, thereby decreasing the magnitude of the electrostatic forces.

The second, short-range regime, between 0.3 and $4 \mathrm{~nm}$, is attributed to the deformation of the bilayer. Most studies on the bilayer report a jump corresponding to the piercing of the bilayer for forces ranging from approximately 3 to $25 \mathrm{nN}$ [71-73]. This jump into contact is observed when the force gradient exceeds the spring constant of the cantilever $k$, which is the case when $k<1 \mathrm{~N} / \mathrm{m}$. Although the force sensitivity can be altered, a high cantilever spring constant $(k=30 \mathrm{~N} / \mathrm{m})$ was chosen for monitoring all the data during the penetration. The force gradient attributed to the layer indentation was measured to be around $10 \mathrm{~N} / \mathrm{m}$ and the force needed to puncture the lipid bilayer is in good agreement with that found in other studies.

The repulsive force in the short-range regime at the contact of the bilayer cannot be easily treated using common elastic mechanical models such as Hertz's theory, or any other models based on continuum mechanics. The heads of the phospholipids are responsible for a crystalline elasticity, i. e., a change of the head-to-head distance (high elasticity but low rupture). The lipid chain behaves like rubber (low elasticity), and this behavior is related to the change of entropy (increase of order when one presses the layer). It results in the property that the cell membranes can be easily deformed in the plane but do not resist the expansion (distance between the headgroup cannot vary more than $2 \%$ ). With our AFM experiments we are examining very small, discrete areas where nanoindentation induces not only macroscopic compression of the bilayer, but also nanoscale structural changes in the bilayer. Therefore, a molecular approach, considering steric forces [42], is preferable.

The exponential increase of the repulsive force likely originates from tail steric repulsion induced by the atomic force microscope tip compressing the bilayer. The tails in the bilayer will resist compression, because compression will increase the local concentration of the alkyl segments, increasing the free energy of the system. The steric forces of compression act on the bilayer until the loading force is close to the yield force, when the tip penetrates through the bilayer. The breakthrough force decreases as the temperature increases, and a DPPC bilayer at elevated temperatures shows similar instability in the force plot as a fluid-phase DOPC bilayer and a fluidphase 1,2-dioleoyl-3-trimethylammonium propane (DOTAP) bilayer [44].

The steric force per unit area that the bilayer exerts on the tip can be modeled using the formalism developed in the work of de Gennes [74]. This model was further developed by Cappella and Dietler [26], where interaction between two polymer surfaces was considered. We modified this formula, considering the interaction of the AFM probe and the bilayer, which gives the formula for the steric repulsion force:

$$
F_{\text {ste }}=100 k T R L \gamma^{3 / 2} \exp (-2 \pi D / L),
$$

where $k$ is the Boltzmann constant, $R$ is the tip radius of curvature, $T$ is the temperature, $\gamma$ is the tail density (inverse of the area per lipid), $D$ is the distance between 
the atomic force microscope tip and the mica surface, and $L$ is the uncompressed bilayer thickness. Similar to the equation for the two interacting polymer surfaces, this formula is valid in the range of distances where $0.2<D / L<0.9$. Since the tip radius is not well defined, it is difficult to estimate the tail density from the fit, but the transition between the two exponential regimes can be a good way to measure the thickness of the bilayer $L$.

The steric forces can, however, be used to determine the thickness of the layer adsorbed at the surface in good agreement with the image cross-section analysis. With the temperature increase, the steric force departs from a pure exponential law owing to the increase of the lateral mobility of the phospholipid tails. The transition between the electrostatic regime and the steric regime where the tip is at the top of the bilayer can be an effective way to estimate the bilayer thickness. At $22{ }^{\circ} \mathrm{C}$, a transition occurs at $D=4 \mathrm{~nm}$, corresponding to a thickness $L=D / 0.9=4.5 \mathrm{~nm}$, which agrees with what is known from the literature $(4.7 \mathrm{~nm})$. With increasing temperature, the magnitude and the range of the steric force decrease contrary to the change in the electrostatic force. The steric force in a fluid phase, at elevated temperatures, is reduced by $50 \%$ compared with that a gel phase, as in Fig. 21.5. This can be explained by the reduction in the tail density with temperature. The tail density is reduced from $1 / 50$ to $1 / 67.1$ (considering an area per lipid of $50 \AA^{2}$ at room temperature and $67.1 \AA^{2}$ at $65^{\circ} \mathrm{C}$ [75-77]), which makes $25 \%$ of difference and therefore a change of $40 \%$ in the force. In a fluid regime, i. e., at $T>50{ }^{\circ} \mathrm{C}$, the shape of the steric part of the force-distance curve starts to depart from an exponential dependence and is similar to the linear abrupt jump observed for a fluid-phase DOTAP bilayer at room temperature. It appears that (21.3) is only valid for a gel-phase DPPC bilayer, where lateral motion of the lipids is lower.

With halothane incorporation (Fig. 21.5, triangles) the force is mostly governed by the bilayer deformation, described by steric forces. The induced phase transition of the DPPC has already been characterized by a decrease in the "effective thickness". The major difference with the temperature-induced phase transition comes from the fact that the incorporation of halothane changes the electrostatic interaction, which was not the case during the melting transition. Our force measurements confirmed that assumption and clearly showed the differences in the physical properties of the bilayer in thin domains, produced by the melting transition and partitioning of halothane.

When the adhesion forces are compared for DPPC and DOPC bilayers, they are similar for the fluid-phase DPPC bilayer at elevated temperatures and the fluidphase DOPC bilayer at room temperature. The unit force $F_{\mathrm{S}}$ for fluid-phase the DPPC bilayer is 0.8 compared with 0.5 for the DOPC bilayer (Table 21.1); the number of unit bonds is 6 for both fluid-phase DPPC and DOPC bilayers. This correlation permits evaluation of a bilayer fluidity and is in a good agreement with imaging data. Therefore, adhesion forces depend on the physical properties of the bilayer, and force analysis gives important information on the nature of the bilayer. In the repulsive part of the force plot using a steric force model also provides an important insight into the properties of the bilayer and ability of lipids to resist compression. Steric forces reduce with the temperature increase for the DPPC bilayer and correlate well with low steric forces for the fluid-phase DOPC bilayer at room temperature. In general, a fluid-phase bilayer can be characterized by the reduced 
repulsive forces, compared with the gel-phase bilayer. The major contribution to this change is provided by changes in steric forces. Lipid compression is much easier in the fluid phase owing to the higher disorder of tails and mobility of lipids, and lower tail density.

Individual bond forces can be estimated for lipid bilayers and changes in binding number can be used as a distinctive feature of the increase of bilayer fluidity. A force analysis revealed that the interaction between the tip and the surface was governed by both the electrostatic forces, for longer range, and the steric forces, at shorter range. Electrostatic repulsion likely originates from an effective charge density at the bilayer-water interface, where zwitterionic headgroups interact with water molecules. The steric forces can be used to determine the thickness of the layer adsorbed at the surface in good agreement with the image cross-section analysis. With the temperature increase, the steric force departs from a pure exponential law owing to the increase of the lateral mobility of the phospholipid tails. The transition between the electrostatic regime and the steric regime where the tip is at the top of the bilayer can be an effective way to estimate the bilayer thickness.

The temperature-induced phase transition of DPPC has been characterized by a decrease in the, "effective thickness" and we also observed similar behavior for bilayers containing halothane [58]. The major difference comes from the fact that the incorporation of halothane changes the electrostatic interaction, which was not the case during the melting transition. Halothane is a small polar molecule and is assumed to increase the polarity of the bilayer at the interface, and has been shown to replace water molecules bound to lipids at the interface. Our force measurements confirmed that assumption and clearly showed the differences in the physical properties of the bilayer in thin domains, produced by the melting transition and partitioning of halothane.

\section{3}

\section{Force Measurements on Pulmonary Surfactant Monolayers in Air}

Pulmonary surfactant is a specific mixture of phospholipids and surfactant-specific proteins. It forms a molecular film at the interface of the hydrated lung epithelium with the air and thereby reduces the surface tension of the interface to near zero. This is required for normal respiration and structural stability of the lung. In adult respiratory distress syndrome (ARDS), surfactant function fails. As a result, the lung is less compliant, the gas exchange area is reduced, and blood oxygenation is strongly decreased. The surfactant film is also the first barrier of airborne particles in polluted air. When inhaled, ultrafine particles with an aerodynamic diameter of $100 \mathrm{~nm}$ or less produce a major health threat. They cross the surfactant film, cross the lung epithelium, and enter the bloodstream. We describe force spectroscopy experiments aimed at a better understanding of surfactant failure in ARDS. Force spectroscopy was used to study the interactions between airborne particles and the surfactant. Particles (modeled by an atomic force microscope tip) interact with the surfactant film before they get into close contact because of the electrical surface potential of the film. This interaction depends on surface potential, which is locally strongly variable, depending on the film composition. 
Fig. 21.6. Supported lipid monolayer and multilayers on mica, transferred from the liquid-air interface by Langmuir-Blodgett deposition; these multilayers are shown on the AFM topography image in Fig. 21.7a

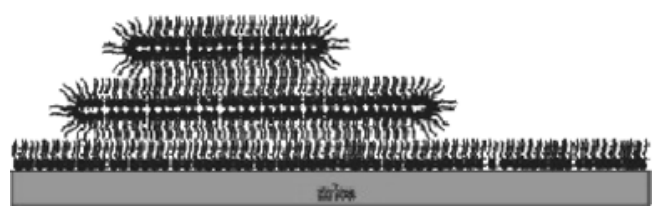

We have shown that the ability of surfactant to reduce surface tension is associated with a distinct molecular architecture of the surfactant film. The functional pulmonary surfactant forms a pattern of molecular monolayer areas and areas of lamellar stacks, cross-linked to the monolayer at the air-aqueous interface (Fig. 21.7a). We recently found that cholesterol in excess of a physiological proportion abolishes surfactant function, and surfactant fails to lower the surface tension upon compression [78]. This failure of function is associated with a change in the molecular architecture [79]. The formation of multilayer stacks, which is a characteristic feature of functional surfactant, does not occur in the presence of excess cholesterol.

We performed atomic force measurements on supported pulmonary surfactant films in air to address the effect of cholesterol on the physical properties of lung surfactant films. We used bovine lipid extract surfactant (BLES). BLES is a hydrophobic extract of bovine lung lavage that differs from natural surfactant in the lack of surfactant-specific proteins SP-A and SP-D and cholesterol. Phosphatidylcholines represent $80 \%$ of its mass with half of the phosphatidylcholines being the disaturated DPPC. Between 5 and 10 mass $\%$ is the negatively charged phosphatidylglycerol, and two hydrophobic surfactant-associated proteins (SP-B, SP-C). BLES in nonbuffered normal saline ( $\mathrm{pH} \mathrm{5-6)} \mathrm{with} \mathrm{a} \mathrm{phospholipid} \mathrm{concentration}$ of $27 \mathrm{mg} / \mathrm{ml}$ was a kind gift from the manufacturer (BLES Biochemical, London, ON, Canada). Cholesterol was purchased from Sigma Chemicals. A solution of 1:1:1 methanol, chloroform, and BLES by volume was first vortexed and then spun at $100 \mathrm{~g}$ for $5 \mathrm{~min}$. The methanol-water phase was discarded and the BLES in chloroform was retained and either 5 or $20 \%$ of cholesterol (by mass) with respect to phospholipids in chloroform was added. Each solution was then dried under $\mathrm{N}_{2}$ and resuspended with Goerke's buffer [140 mM NaCl, $10 \mathrm{mM} \mathrm{N}$-(2hydroxyethyl)piperazine- $N^{\prime}$-ethanesulfonic acid and $2.5 \mathrm{mM} \mathrm{CaCl}_{2} ; \mathrm{pH}$ 6.9] to obtain an aqueous suspension of BLES and cholesterol at a concentration of $27 \mathrm{mg} / \mathrm{ml}$ phospholipids. BLES solutions were spread at the air-liquid interface. Supported planar monolayers on mica were prepared using Langmuir-Blodgett technique and transferred on mica support when films were compressed to a surface tension of $25 \mathrm{mN} / \mathrm{m}$. Supported films were imaged in air using a NanoWizard atomic force microscope from JPK Instruments, Germany. All measurements were performed at $25^{\circ} \mathrm{C}$, over five different velocities, collecting each time ten force curves. Silicon cantilevers from Micromash, Spain, were used with cantilever spring constants of 0.6 and $0.7 \mathrm{~N} / \mathrm{m}$ as determined by a thermofluctuation method using JPK SPM software. Forces were measured on several different structural areas of the film, by positioning the atomic force microscope tip after the image had been collected. 


\subsection{1}

\section{Adhesion Measurements: Monolayer Stiffness and Function}

Atomic force measurements helped us to elucidate how the loss of mechanical stability is related to the local mechanical properties of the film. Films containing 5 and $20 \% \mathrm{w} / \mathrm{w}$ cholesterol were compared. The presence of $20 \%$ cholesterol in BLES resulted in a decrease of the observed adhesive interaction, and an increase in the rigidity of the film. The force measurements were performed both on the monolayer area and on a first bilayer adjacent to the monolayer. The typical height of the bilayer area is $4.5 \mathrm{~nm}$, and that of the monolayer is $2.5 \mathrm{~nm}$. Typical areas where forces were measured are shown in Fig. 21.7a. For BLES with $20 \%$ cholesterol, only a monolayer was present and was used for force measurements (Fig. 21.7a, point 1).

BLES with 5\% cholesterol at the bilayer area has an adhesion of $60 \mathrm{nN}$, which is higher than that of the monolayer. BLES with $20 \%$ cholesterol has a lower adhesion than the BLES monolayer with 5\% cholesterol. It is interesting that the "single force" $F_{\mathrm{s}}$ was slightly lower in the presence of $20 \%$ cholesterol, $3 \mathrm{nN}$ compared with $4 \mathrm{nN}$ with $5 \%$ cholesterol, but the number of bonds decreased significantly from 15 and 10 to 7 (Table 21.1 [79]). Therefore, the presence of $20 \%$ cholesterol decreases the fluidity of the monolayer.

A force histogram (Fig. 21.8) was used to estimate the single force $F_{\mathrm{s}}$ and the number of bonds $n$. It is interesting that $F_{\mathrm{s}}$ was slightly lower in the presence of $20 \%$ cholesterol, $3 \mathrm{nN}$ compared with $4 \mathrm{nN}$ with $5 \%$ cholesterol, but $n$ decreased significantly from 15 and 10 to 7 (Table 21.2). Therefore, the presence of $20 \%$ cholesterol decreases the fluidity of the monolayer.

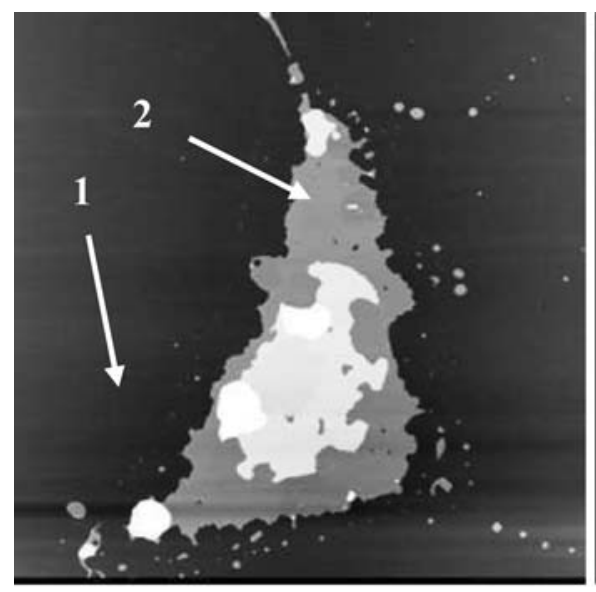

A

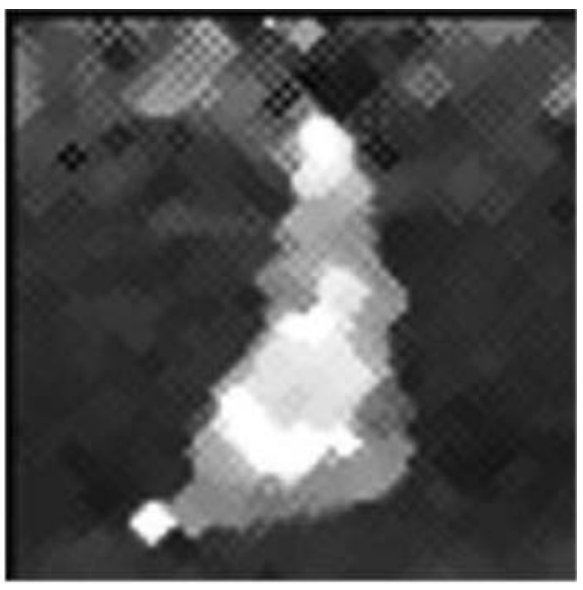

B

Fig. 21.7. A AFM topography image of supported bovine lipid extract surfactant (BLES) film, scan area $15 \mu \mathrm{m}$ by $15 \mu \mathrm{m}$. B Adhesion force map image 64 by 64, collected on the same area. The adhesion force map was collected while forces of interaction at each of 64 by 64 points were measured. The adhesion peak of the retrace plot was used to generate the adhesion force map image. 1 area of a typical monolayer; 2 area of a typical bilayer, adjacent to a monolayer 
Table 21.2. Experimental adhesion force $F_{\text {adh }}$ and its standard error $\sigma$ on a bovine lipid extract surfactant (BLES) bilayer with with 5\% cholesterol, a BLES monolayer with 5\% cholesterol, and a BLES monolayer with $20 \%$ cholesterol, measured at room temperature; calculation of the number of bonds $n$ and the mean force of a single bond $F_{\mathrm{s}}$. Rate $200 \mathrm{~nm} / \mathrm{s}$, load $20 \mathrm{nN}$. Adhesion force on pure mica does not exceed $1.5 \mathrm{nN}$

\begin{tabular}{llll}
\hline & $\begin{array}{l}\text { BLES 20\% cholesterol } \\
\text { monolayer }\end{array}$ & $\begin{array}{l}\text { BLES 5\% } \\
\text { cholesterol monolayer }\end{array}$ & $\begin{array}{l}\text { BLES 5\% } \\
\text { cholesterol bilayer }\end{array}$ \\
\hline$F_{\text {adh }}(\mathrm{nN})$ & 20 & 40 & 60 \\
$\sigma(\mathrm{nN})$ & 8 & 12 & 15 \\
$F_{\mathrm{S}}(\mathrm{nN})$ & 3 & 4 & 4 \\
$n$ & 7 & 10 & 15 \\
\hline
\end{tabular}

Fig. 21.8. Distribution of adhesion force, $F_{\text {adh }}$, in air: a BLES monolayer + $20 \%$ cholesterol; b BLES monolayer $+5 \%$ cholesterol; c BLES bilayer + $5 \%$ cholesterol
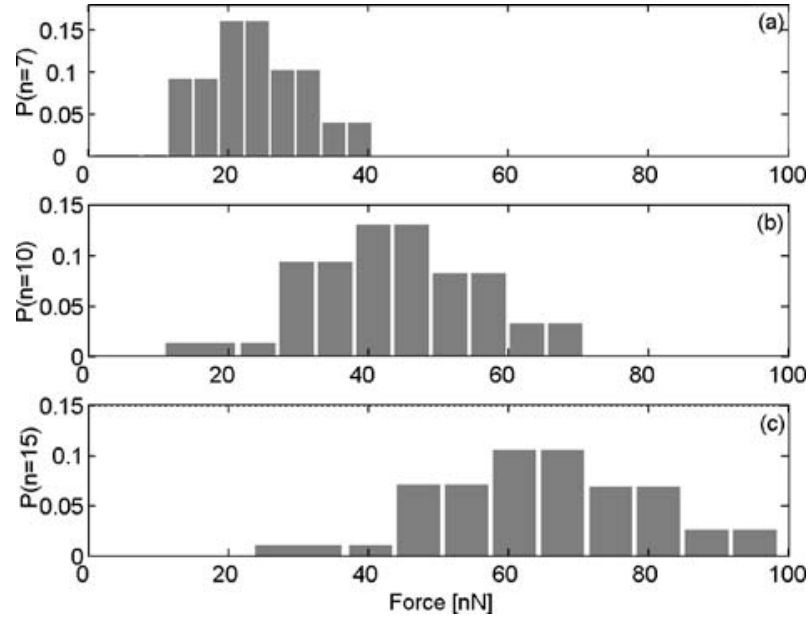

The increase in the adhesion force for a bilayer as compared with a monolayer may be understood by an increase in the contact area between the AFM probe and the sample at a given load. BLES with $20 \%$ cholesterol shows the lowest adhesion force, compared with monolayer and multilayer areas of BLES with 5\% cholesterol. This indicates that adhesion properties of the film were considerably altered by the incorporation of $20 \%$ cholesterol, decreasing adhesive interaction, and increasing the rigidity of the film. The lipid molecules are not mobile enough in the presence of $20 \%$ cholesterol and cannot be easily rearranged around the tip to increase the contact area and adhesion force. The increased rigidity of the film and decreased adhesion that we observed may play an important role in preventing the monolayer-bilayer conversion.

\subsection{2}

\section{Repulsive Forces: The Interaction of Charged Airborne Particles with Surfactant}

The repulsive forces (Fig. 21.9) observed in air between the lipids film and the AFM probe were analyzed using two theoretical models for electrostatic interaction. The atomic force microscope tip was modeled once as a point charge and once as 
Fig. 21.9. Electrostatic force measured in air on BLES film with $0 \%$ cholesterol (top) and with $20 \%$ cholesterol (bottom). Experimental data are shown by dots and theoretical fits with the point-charge model are drawn in solid lines

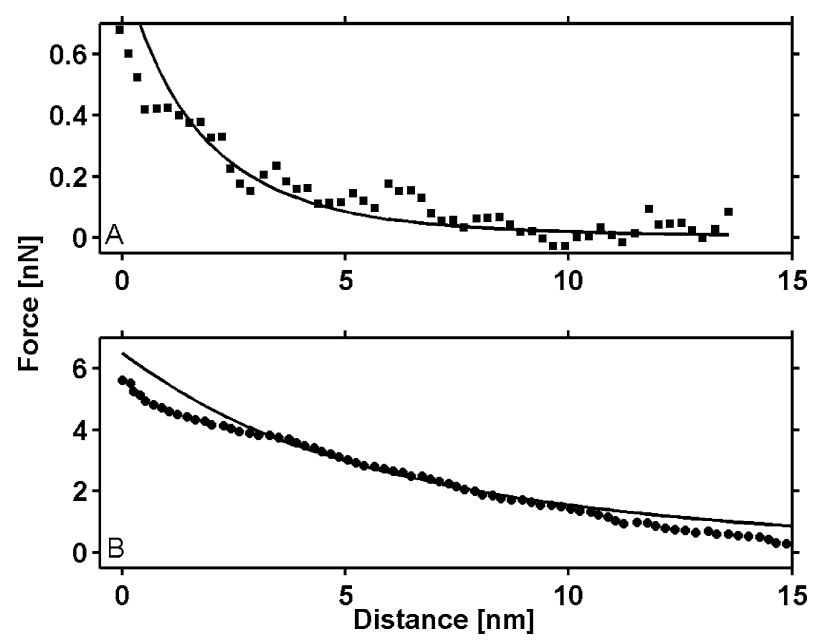

a charged semisphere. Here $q$ is a charge of the atomic force microscope tip, $k$ is a constant, $\varepsilon$ is the dielectric permittivity of the medium, and $r$ is the separation distance between the tip and the medium. The supported BLES surfactant monolayer is composed of lipid molecules, which are organized and oriented in such a way that the dipole moment is directed along the surface normal. Therefore, the patch of the monolayer can be treated as two charged planes, each having charge $Q_{+}$and $Q_{-}$and surface charge density $\sigma_{+}$and $\sigma_{-}$, with the separation distance $\delta_{0}$, which corresponds to the thickness of the monolayer and is equal to $2.5 \mathrm{~nm}$. The surface charge density of the sample, $\sigma$, is related to the surface potential $V$, which we have determined experimentally using Kelvin probe force microscopy [80,81] and used here as a parameter.

In the case of a point charge, the total electrostatic force acting on $q$ is given by

$$
F=\frac{V_{\mathrm{q}}}{2 \delta_{0} A_{0}}\left(\frac{\beta}{\sqrt{1+\beta^{2}}}-\frac{\alpha}{\sqrt{1+\alpha^{2}}}\right),
$$

where

$$
\begin{aligned}
\frac{D}{R_{0}} & =\alpha, \\
\frac{D+\delta_{0}}{R_{0}} & =\beta .
\end{aligned}
$$

For the semisphere model, the force acting on the semisphere tip with a radius $R_{\text {so }}$ will be

$$
F_{\mathrm{s}}=\frac{V_{\mathrm{q}}}{2 \delta_{0} A_{0}}\left(\sqrt{1+\beta_{1}^{2}}-\sqrt{1+\alpha_{1}^{2}}-\sqrt{1+\left(\beta_{1}-\gamma\right)^{2}}+\sqrt{1+\left(\alpha_{1}-\gamma\right)^{2}}\right),
$$


where

$$
\begin{aligned}
\frac{D+R_{\mathrm{S} 0}}{R_{0}} & =\alpha_{1}, \\
\frac{D+R_{\mathrm{S} 0}+\delta_{0}}{R_{0}} & =\beta_{1}, \\
\frac{R_{\mathrm{S} 0}}{R_{0}} & =\gamma .
\end{aligned}
$$

Kelvin probe microscopy was used [81] to determine the surface potential of the lipid layer in air. Cholesterol induces a nonuniform distribution of the electric surface potential even at small physiological concentrations, showing nanoscale domains in the Kelvin image, while the AFM topography image remains uniform. Such domains vary in surface potential from $-0.6 \mathrm{~V}$, measured for $0 \%$ cholesterol, to $-0.4 \mathrm{~V}$, at $20 \%$ cholesterol.

Both models we used fit the experimental data well, showing the presence of electrostatic interactions in the net force measured between the tip and the sample. To better fit the experimental data we varied the charge on the atomic force microscope tip and the size of the charged disks, $R_{0}$, which experimentally corresponds to the size of domains already observed with both AFM and Kelvin probe force microscopy imaging. We modeled the AFM probe also with a charged sphere; in this model the effective charge depends on the radius of the tip. Samples of BLES with $20 \%$ cholesterol exhibit stronger electrostatic repulsion at longer distances, for all models used.

Interestingly, the electrostatic force was found to be more sensitive to the size of these domains than to the changes in the surface potential observed owing to the effect of cholesterol. The experimental data and the theoretical fit correlate well and both revealed that the size of the domains is approximately 5 times larger for the film containing $20 \%$ cholesterol than that for the film with no cholesterol present. This confirms the important role of cholesterol-it produced small domains which differ by electrostatic potential, initially invisible with AFM, which causes the increase in the total electrostatic force. These findings correlate with the results of molecular dynamics simulations [82], where cholesterol was shown to order individual lipids adjacent to cholesterol molecules, but abolished the long-range cooperativity between lipids, characteristic for the gel phase with true latticelike spatial order, and altered the lipid electrostatic potential [83]. In the presence of cholesterol [84] it was shown that the membrane electrostatic potential has a much larger variance depending on the distance along the bilayer normal, compared with that for a pure DPPC bilayer.

\section{4}

\section{Interaction Forces Measured on Lung Epithelial Cells in Buffer}

Elevated levels of ultrafine particles in air pollution are associated with increased morbidity and mortality [85]. Unlike larger particles, ultrafine particles reach the peripheral lung and are able to cross the various barriers of the lung, including 
the lipid-protein layer of pulmonary surfactant at the air-alveolar interface and the cell membranes of the epithelial and endothelial cells underneath and reach the bloodstream [86-89]. The toxicity of inhaled nanoparticles entering the body through the lung is thought to be initially defined by the electrostatic and adhesive interaction of the particles with the wall of the lung, and lung epithelial cells. Because of their substantial uptake, ultrafine particles are also of great interest for delivery of drugs to the peripheral lung and the body. Despite the importance of the early penetration steps in the biological effects of inhaled particles, the nature of the interactions with the various barriers of the lung remain poorly understood. A detailed study, therefore, promises substantial progress in the understanding of the health threat posed by fine and ultrafine air pollutants and the effectiveness of drug delivery by aerosols. The adhesive interaction includes electrostatic, van der Waals, steric, and hydrophobic forces as well as the line tension between particle, cell, and aqueous medium and depends on the elastic and plastic properties of the cell. The free energy due to these interactions will be minimized upon particle wetting by the lining layer. The lower the surface free energy of the particle, the less it will be wetted by the cell. A thermodynamic model using the "wettability criterion" was indeed successful in predicting passive particle uptake by cells [90]. Another thermodynamic analysis combined with a molecular dynamics simulation found negative line tension values for nanometer-sized particles, but positive values for those an order of magnitude larger [91]. The engulfment of particles in the nanometer range by unbalanced capillary forces was first suggested by Shanahan [92].

We demonstrated that the initial thermodynamic aspects and the time course of the uptake of nanoparticles by lung epithelial cells can be studied using AFM [93] in the force measurement mode. We investigated the first steps of the interaction of nanoparticles with lung epithelial cells using the atomic force microscope as a force apparatus. The apex of theatomic force microscope tip can be used as a model of the nanoparticles, thereby enabling the monitoring of the interaction forces between the nanoparticles and the cell over time. The adhesion force and the work of adhesion have been investigated and correlated with the mechanical properties of the cell.

\subsection{1}

\section{Cell Culture/Force Measurement Setup}

Lung epithelial cells, type II, were isolated from male, pathogen-free SpragueDawley rats weighing $150-250 \mathrm{~g}$. The cells were cultured at $6 \times 10^{8}$ cells per milliliter in Dulbecco's modified Eagle's medium supplemented with 1\% Lglutamine, containing $10 \%$ heat-inactivated fetal bovine serum, and $300 \mu \mathrm{l}$ of a penicillin-gentamycin solution in $\gamma$-irradiated culture dishes. The cells were grown on light microscope glass cover slips. The cells were kept in an incubator under 5\% $\mathrm{CO}_{2}$ and $a t 37^{\circ} \mathrm{C}$ until they created a monolayer with approximately $80 \%$ of confluence (4-6 days). At this stage, the phenotype of the lung epithelial cells had changed from type II to type I in that the cells were spread out flat on the interface and ceased to produce surfactant.

Lung epithelial cells were first imaged using a light microscope (Zeiss Axiovert 200). For force spectroscopy, a medium-covered cover slip with the cells was mounted in the liquid cell of the atomic force microscope. The setup used allows for 
the observation of the sample and the atomic force microscope cantilever across the cover slip from below by an inverted light microscope at the same time as force spectroscopy (or AFM imaging) is performed. The tip of the atomic force microscope was thus placed over a suitable cell and cell location was under light-microscope control (Fig. 21.10a). Figure 21.10b shows a typical force scan where the cantilever deflection was monitored as a function of the sample position.

At first, the probe approached the cell with the lever undeflected (horizontal line of the force trace), indicating no interaction. The tip then made contact with the sample and became deformed. Forces of interaction between the probe and the cell were then measured in medium at $35^{\circ} \mathrm{C}$, by approach of the tip to the cell interface until a preset load was reached (trace of force curve). The force was acquired as a function of the tip-sample separation $h$. After the tip has come into contact with the cell, $h$ becomes the penetration depth for the tip into the cell. Separate force curves were acquired for a preset load of 100,200 , and $300 \mathrm{pN}$. The tip was now kept in contact with the cell for a predetermined time (delay time), while the preset load was kept constant under feedback control (i.e., when the load decreased, the tip was moved forward). We varied the delay time from 0 to $1800 \mathrm{~s}$. Thereafter, the tip was retracted and the adhesion observed. Cantilevers (Micromash), with a spring constant of $57 \mathrm{mN} / \mathrm{m}$, and pyramidal silicon nitride tips were used (tip height $2.9 \mathrm{~nm}$, tip radius less than $20 \mathrm{~nm}$ (typical $10 \mathrm{~nm}$ ), tip angle (face to face) $25-45^{\circ}$ (top to around $300 \mathrm{~nm}$ down). Cells were kept in the medium at all times. The spring constant for each cantilever was measured before and after the experiment using the procedures implemented by the manufacturer of the atomic force microscope

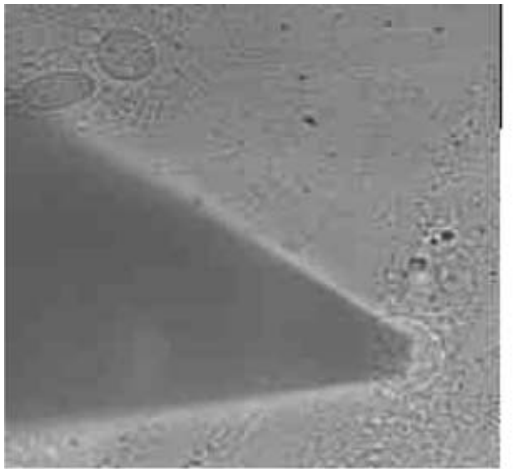

A

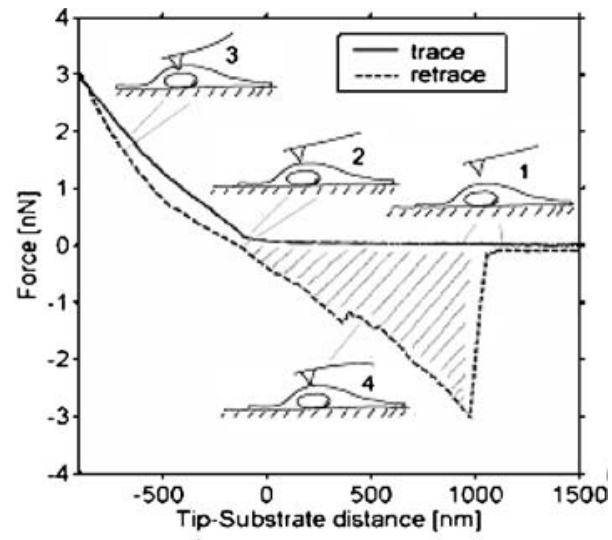

Fig. 21.10. Optical microscopy image of epithelial type II cells and atomic force microscope cantilever. For force spectroscopy, an atomic force microscope tip was placed over the central region of a cell. Trace and retrace force curves were measured on a cell. Force versus tip-sample separation is shown in $\mathbf{b}$. The approach part (trace) reveals how the cell deforms upon contact by the tip. The retrace or withdraw part of the curve reveals a large adhesion peak which is often split in multiple peaks. The sketches explain the physical situation for each part of the curve. The hatched area denotes the work of adhesion (adhesion energy) between the tip and the cell. The atomic force microscope tip was allowed to stay in contact for various times (delay time) in position $\$ 3$ 
(thermal noise method). The sensitivity and the spring constant were found to be not different before and after the experiment.

Forces of interaction between the probe and the cell were then measured in the medium at $35^{\circ} \mathrm{C}$.

\subsection{2}

\section{Mechanical Properties}

The approach part of the force curve was used to determine the penetration depth of the tip into the cell as well as the mechanical properties of the sample. Reviews on this topic are given in $[94,95]$.The measurement of the penetration depth will depend on the accurate determination of the contact point between the tip and the cell. A cantilever spring constant lower than or similar to that of the cell is preferable. In this case, we can assume that the contact point corresponds in the trace force curve to the first upward deflection. After the tip has come into the contact with the cell, $D$ becomes the penetration depth $h$ of the tip into the cell. To evaluate the mechanical properties of the sample from the approach force profile, the Sneddon model was employed $[26,96]$. Note that the use of macroscopic physical law should be reserved for homogeneous bulk materials. Young's modulus should be viewed as an effective parameter to analyze the trend of the in-plane organization of supramolecular structures. The effective parameter can differ from one experiment to another. A more sophisticated approach was to model the mechanical response of the cell membrane explicitly taking into account the mechanics of the cell membrane and that of the underlying cytoskeleton [97]. As the penetration depth $h$ can reach $1 \mu \mathrm{m}$, the tip shape must be considered as a function of $h$. The effective Young's modulus, $E^{\prime}$, was estimated point by point for $h$ considering the three regions determined by the geometry of the tip [98].

For $h$ between 0 and $10 \mathrm{~nm}$, the AFM probe was considered as a semisphere with a radius $R$ of $10 \mathrm{~nm}$ :

$$
E_{\text {sphere }}=\frac{3}{4 \sqrt{R}} \frac{F}{h^{3 / 2}} .
$$

For further penetration, the tip profile was viewed as a cone whose half angle varies linearly from $\alpha_{1}=25^{\circ}$ at $h=10 \mathrm{~nm}$ to $\alpha_{2}=45^{\circ}$ when $h$ reaches $300 \mathrm{~nm}$ :

$$
E_{\text {cone }}=\frac{\sqrt{2}}{\tan \alpha} \frac{F}{h^{2}} .
$$

For an indentation deeper than $300 \mathrm{~nm}, \alpha$ is considered constant to $\alpha_{2}$.

Figure 21.11 shows the change in the Young's modulus when increasing the penetration depth $h$. At first, the tip experienced a layer of increasing stiffness to a penetration depth of about $100 \mathrm{~nm}$, at which point the resistance of the cell to penetration reached a maximum. Thereafter the cell became more compliant again. The stiff layer may represent the outer layer of the cytoskeleton anchored to the cell membrane. The Young's modulus of the membrane can be thought of as the surface tension of the membrane $(0.5 \mathrm{~N} / \mathrm{m})$ divided by the thickness of the membrane (around $10 \mathrm{~nm}$ ), i.e., a pressure of around $50 \mathrm{MPa}$. The lipid head is responsible 
Fig. 21.11. When analyzing the approach curve, one can determine the Young's modulus of the sample surface. Young's modulus, which gives a measure of cell elasticity in our case, was found to depend on indentation depth, showing two distinctive parts of the plot: when the peripheral part of the cell was penetrated, up to about $100 \mathrm{~nm}$ into the cell, the cell became increasingly stiffer. The stiffness then dropped sharply upon further penetration until it leveled out after penetration to about $200 \mathrm{~nm}$ deep

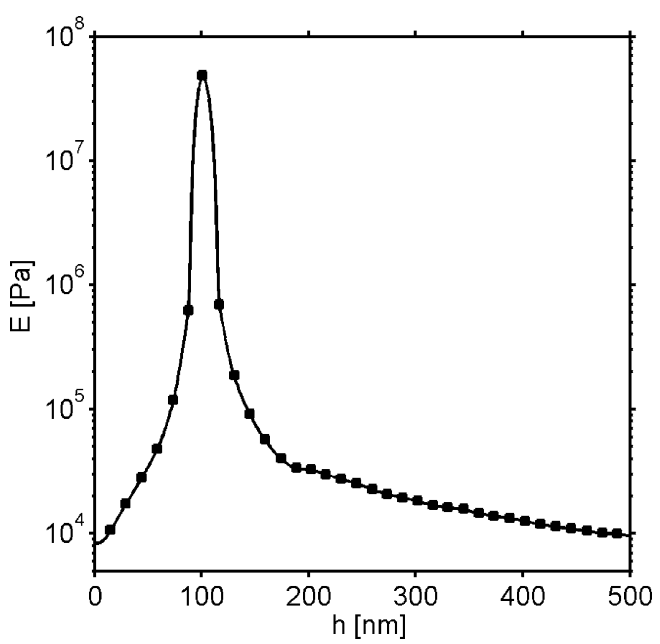

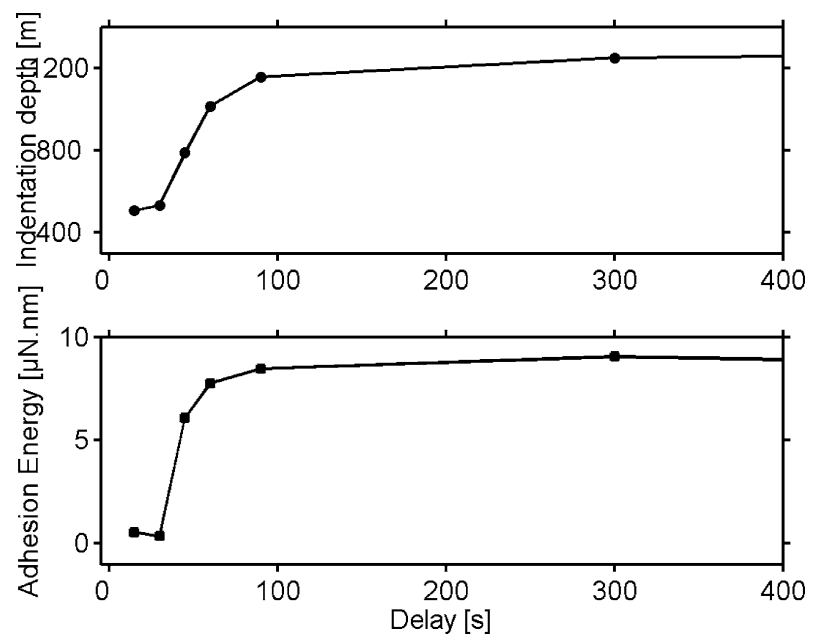

Fig. 21.12. Top: Indentation depth as a function of time. The load was kept constant at $200 \mathrm{pN}$. At time zero, the indentation was about $500 \mathrm{~nm}$. Over time, the tip first penetrated rapidly and then slowly from 500 to $1300 \mathrm{~nm}$. This is evidence of a time-dependent response of the cell to the tip. Bottom: Adhesion energy as a function of time. The tip was kept in contact with the cell at a constant load of $200 \mathrm{pN}$. The longer the tip stays in contact with the cell, the larger the adhesion that is observed. Statistical analysis shows that considerable changes occur during the first $100 \mathrm{~s}$, after which the mean value of the adhesion energy no longer changes

for crystalline elasticity, i.e., the change in the head-to-head distance can change (high elasticity but low rupture). The lipid chain behaves with rubber elasticity (low elasticity), which is related to the change of entropy (increase of order upon pressure). It results in the properties that the cell membranes can be easily deformed in the plane but do not resist the expansion (increase between the headgroup cannot vary more than $2 \%$ ). 
Further measurements of the Young's modulus of cells have been performed with human platelet cells $(E=1-50 \mathrm{kPa})$ [99], cardiac cells $(E=100 \mathrm{kPa})$, skeletal muscle cells $(E=25 \mathrm{kPa})$, and endothelial cells $(1-7 \mathrm{kPa})$ [100].

The analysis of the forced indentation of the epithelial cells by the tip reveals the fate of a nanoparticle coming into contact with the lung's epithelium and then being actively loaded onto a cell. This situation will indeed occur in the lung. The lung's epithelium towards the air is covered by a thin aqueous layer and a molecular film of pulmonary surfactant at the air-water interface. Transmission electron microscopy of lung thin sections as well as scanning electron microscopy of the lung have indeed shown that particles trapped between the surfactant layer and the epithelial cells strongly deform the cells $[101,102]$.

The penetration depth was also investigated as a function of the contact time between the tip and the cell at a given load. Figure 21.12 shows that the tip first penetrated the cell down to $500 \mathrm{~nm}$ in less than $100 \mathrm{~s}$. Equilibrium was reached for approximately 1- $\mu \mathrm{m}$ depth (around half of the cell thickness). We assume that the tip is either actively taken up or the cell rearranges its plasma membrane and the cytoskeleton elements to accommodate the tip.

\subsubsection{1}

\section{Adhesion Work}

After the initial indentation, the tip was kept loaded onto the cell with $1 \mathrm{nN}$ for preset times ranging from 0 to $900 \mathrm{~s}$ under feedback control. Thereafter, the tip was retracted and the tip adhesion was acquired.

The retrace curve was used to calculate the adhesion energy $\Gamma$ between the tip and the cell:

$$
\Gamma=\sum_{h_{1}}^{h_{2}} F_{\text {retrace }}\left(h_{i}\right)\left(h_{i+1}-h_{i}\right) .
$$

$F_{\text {retrace }}\left(h_{i}\right)$ is the adhesion force at $h_{i}$. The point of the initial contact between the tip and the cell surface, $h_{1}$, was determined from the approach part of the force curve; $h_{2}$ corresponds to the last jump out of contact.

Upon the penetration into the cell, the adhesion energy also increases. This indicates that the tip becomes engulfed by the membrane and the area of interaction increases (Fig. 21.12). Our results suggest that the process of particle uptake by lung epithelial cells occurs over approximately $100 \mathrm{~s}$. This appears to be the time necessary for the nanoparticle to increase its surface area in contact with the cell and, hence, its adhesive interaction. Other authors have also shown [103] that the increase in adhesion between a polystyrene sphere to mica with increasing load or contact time is due to the plastic deformation. Load dependence and contact time dependence also indicate plastic and viscoelastic deformation [104]. A linear dependence of adhesion force on the reduced radius $R_{\text {eff }}=R_{1} R_{2} /\left(R_{1}+R_{2}\right)$, where $R_{1}$ and $R_{2}$ are the radii of the two particles, was found by Heim et al. [105].

Particles of 50-100 nm in diameter are not phagocytosed [106, 107]. They enter cells in the absence of clathrin and caveolin, associated with active uptake. Even 
Fig. 21.13. Correlation between the indentation depth (Fig. 21.12, top) and the adhesion energy (Fig. 21.12, bottom) for all the measurements (around 100 points). The deeper the indentation, the higher is the adhesion energy

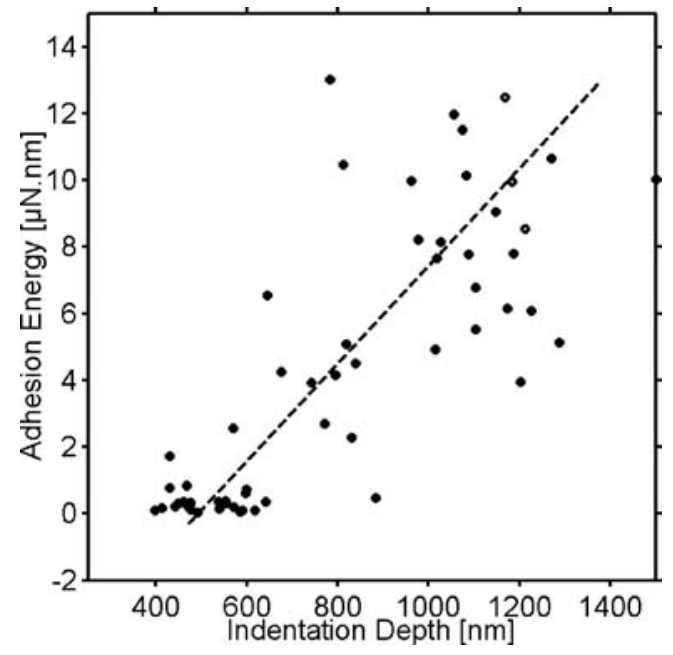

when an actin-based mechanism has been ruled out [107], nanoparticles inside a cell are not surrounded by membrane and, hence, are not taken up through vesicle formation.

The AFM investigation suggests the adhesive interaction in particle uptake can now be measured by our approach and used as a significant parameter test. Particles with different surface properties and different geometries can be mimicked by modified atomic force microscope tips. The adhesive interaction of a particle with the plasma membrane in the lung depends also on the particle history. Airborne particles, after crossing a surfactant layer at the air-water interface of a cell culture dish, have been shown to be more readily taken up than particles added to the media [108]. They may have become coated with a film of pulmonary surfactant and, as a consequence, have penetrated the epithelial cells differently. Whether this is related to a change in adhesive interaction can be tested by coating atomic force microscope tips with surfactant.

\section{5}

\section{Conclusions}

Measurements of forces with the atomic force microscope can determine the local changes in the mechanical and electrical properties induced by the absorption of proteins within lipid monolayers or bilayers. Such studies have offered new perspectives in the identification and characterization of the pulmonary surfactant films in air, model membranes in liquid media, as well as cell membranes in media.

In air, the adhesion forces of particles are known to be 10 times higher than in aqueous media. For a given applied load, the differences in the magnitude of the force do not depend on the contact area, which is similar in air or in a liquid. In fact, the van der Waals forces are screened under a liquid, thereby reducing the total adhesion forces. However, the adhesion forces can be modulated by varying the 
contact area between the particle and the layer. We have shown that the temperature or the anesthetic incorporation can largely modify the fluidity of the membrane.

Using the AFM probe as a nanoparticle model is an idea to evaluate the ability of the particle to interact with the cell. Although many kinds of force curve cannot yet be interpreted, for instance, the role of the hydrophobic attraction between tails, our work is a starting point to understand how the particle can penetrate through the lipid layer. For example, the nonnegligible role of the electrical forces in air has been proved and the electrical potential of the layer is of importance in the adsorption process involving the electrical barrier.

The mechanical properties of the cell, and in general the plastic deformation, have been investigated. The role of the membrane as a mechanical barrier is clearly observed by measuring the Young's modulus as a function of the penetration depth. Those properties depend on many parameters: the particle speed, the particle size, the applied load. Also, the duration of the contact is crucial in the incorporation ability of the particles. The complexity of the phenomenon cannot of course be satisfactorily and fully described, but some empirical trend can be locally and statistically defined.

Acknowledgements. The authors gratefully acknowledge financial support from the National Sciences and Engineering Research Council of Canada, the Canadian Institute for Health Research, the University of Calgary, and the University of Burgundy and also thank C. Le Grimellec and M. Moskovits for critical reading of the manuscript and helpful discussion.

\section{References}

1. Cullis PR, Dekruijf B (1979) Biochim Biophys Acta 559:399-420

2. Seelig J, Seelig A (1980) Q Rev Biophys 13:19-61

3. Brown DA, London E (1998) J Membr Biol 164:103-114

4. Nagle JF, Tristram-Nagle S (2000) Biochim Biophys Acta Rev Biomembr 1469:159-195

5. Bloom M, Evans E, Mouritsen OG (1991) Q Rev Biophys 24:293-397

6. Brown DA, London E (2000) J Biol Chem 275:17221-17224

7. Brown DA, London E (1998) Annu Rev Cell Devel Biol 14:111-136

8. Vangolde LMG, Batenburg JJ, Robertson B (1988) Physiol Rev 68:374-455

9. Lewis JF, Jobe AH (1993) Am Rev Respir Dis 147:218-233

10. Possmayer F (1988) Am Rev Respir Dis 138:990-998

11. Vedhuizen R, Nag K, Orgeig S, Possmayer F (1998) Biochim Biophys Acta Mol Basis Dis 140:890-108

12. Rooney SA, Young SL, Mendelson CR (1994) FASEB J 8:957-967

13. Tamm LK, McConnell HM (1985) Biophys J 47:105-113

14. Tien HT, Ottova AL (1998) Electrochim Acta 43:3587-3610

15. Heyse S, Ernst OP, Dienes Z (1998) Biochemistry 37:507-522

16. Muller RH, Mader K, Gohla S (2000) Eur J Pharma Biopharma 50:161-177

17. Leonenko ZV, Carnini A, Cramb DT (2000) Biochim Biophys Acta 1509:134-140

18. Zasadzinski J, Helm CA, Longo ML, Weisenhorn AL, Gould SAC, Hansma PK (1991) Biophys J 59:755-760

19. Hui SW (1995) Mol Membr Biol 12:45-50

20. Singh S, Keller DJ (1991) Biophys J 60:1401-1410

21. Beckmann M, Nollert P, Kolb HA (1998) J Membr Biol 161:227-233 
22. Han W, Lindsay SM, Jing T (1996) Appl Phys Lett 69:4111-4113

23. Han W, Lindsay SM (1998) Appl Phys Lett 72:1656-1658

24. Fasolka MJ, Mayes AM (2001) Annu Rev Mater Res 31:323-355

25. Lee GU, Kidwell DA, Colton RJ (1994) Langmuir 10:354-357

26. Cappella B, Dietler G (1999) Surf Sci Rep 34:1-104

27. Senden TJ, Drummond CJ, Ke'kicheff P (1994) Langmuir 10:358-362

28. Cleveland JP, Schäffer TE, Hansma PK (1995) Phys Rev B 52:8692-8695

29. Israelachvili J (2005) Q Rev Biophys 38:331-337

30. Livadaru L, Kovalenko A (2006) Nano Lett 6:78

31. Biggs S (1995) Langmuir 11:156-162

32. Dufrene YF, Boland T, Schneider JW, Barger WR, Lee GU (1998) Faraday Discuss 111:79

33. Finot E, Lesniewska E, Mutin J-C, Goudonnet J-P (1999) J Chem Phys 111:6590-6598

34. Finot E, Lesniewska E, Mutin J-C, Goudonnet J-P (2000) Langmuir 16:4237-4244

35. Finot E, Lesniewska E, Mutin J-C, Goudonnet J-P (2000) Appl Surf Science 161:316-322

36. Butt HJ, Cappella B, Kappl M (2005) Surf Sci Rep 59:1-152

37. Finot E, S Roualdes, Kirchner M, Rouessac V, Berjoan R, Durand J, Goudonnet J-P, Cot L (2002) Appl Surf Sci 187:326-338

38. Mollica V, Relini A, Rolandi R, Bolognesi M, Gliozzi A (2000) Eur Phys J E 3:315

39. Van EW der Vegte, Hadziioannou G (1997) Langmuir 13:4357-4368

40. Dimitriadis EK, Horkay F, Maresca J, Kachar B, Chadwick RS (2002) Biophys J 82:27982810

41. Legay G, Finot E, Meunier-Prest R, Cherkaoui-Malki M, Latruffe N, Dereux A (2005) Biosens Bioelectron 21:627-636

42. Franz V, Loi S, Muller H, Bamberg E, Butt H-J (2002) Colloids Surf B 23:191-200

43. Butt HJ, Franz V (2002) Phys Rev E 66:1601

44. Loi S, Sun G, Franz V, Butt HJ (2002) Phys Rev E 66:1602

45. Tenchov B, Koynova R, Rapp G (2001) Biophys J 80:1873-1890

46. Meyer HW, Semmler K, Rettig W, Pohle W, Ulrich AS, Grage S, Selle C, Quinn PJ (2000) Chem Phys Lipids 105:149-166

47. Bizzotto D, Nelson A (1998) Langmuir 14:6269-6273

48. Tamm LK (1985) Biol Chem Hoppe-Seyler 366:859-859

49. Kalb E, Frey S, Tamm LK (1992) Biochim Biophys Acta 1103:307-316

50. Nollert P, Kiefer H, Jahnig F (1995) Biophys J 69:1447-1455

51. Exerowa D (2002) Adv Colloid Interface Sci 96:75-100

52. Ebel H, Grabitz P, Heimburg T (2001) J Phys Chem B 105:7353-7360

53. Giocondi MC, Vie V, Lesniewska E, Milhiet PE, Zinke-Allmang M, Le C Grimellec (2001) Langmuir 17:1653-1659

54. Leonenko Z, Cramb D (2002) Nano Lett 2:305-309

55. Leonenko ZV, Merkle DD, Lees-Miller SP (2002) Langmuir 18:4873-4884

56. Tokumasu F, Jin AJ, Dvorak JA (2002) J Electron Microsc 51:1-9

57. Leonenko Z, Cramb D (2004) Can J Chem 82:1-11

58. Leonenko Z, Finot E, Cramb D (2006) Biochim Biophys Acta Biomembr 1758:487-492

59. Leonenko ZV, Carnini A, Cramb DT (2000) Biochim Biophys Acta 1509:131-147

60. Brian AA, McConnell HM (1984) Proc Natl Acad Sci USA 81:6159-6163

61. Leonenko ZV, Finot E, Ma H, Dahms T, Cramb DT (2004) Biophys J 86:3783-3793

62. Shamrakov LG, Leonenko Z, Finot E, Cramb DT (2006) Aust J Chem 59:386-389

63. Biltonen RL, Lichtenberg D (1993) Chem Phys Lipids 64:129-142

64. Yang J, Appleyard J (2000) J of Phys Chem B 104:8097-8100

65. Binnig G, Quate CF, Gerber C (1986) Phys Rev Lett 56:930-933

66. Israelachvili J (1992) Intermolecular and surface forces. Academic, New York

67. Williams JM, Han T, Beebe TP (1996) Langmuir 12:1291-1295 
68. Marrink SJ, Berger O, Tieleman P (1998) Biophys J 74:931-943

69. Evans K, Evans MF, Laker MF (1995) Ann Clin Biochem 32:261-280

70. Butt HJ (1991) Biophys J 60:1438-1444

71. Butt HJ, Franz V (2002) Phys Rev E 66:1601

72. Carnini A, Phillips HA, Shamrakov LG, Cramb DT (2004) Can J Chem 82:1139-1149

73. Garcia-Manyes S, Oncins G, Sanz F (2005) Biophys J 89:4261-4274

74. de Gennes PG (1987) Adv Colloid Interface Sci 27:189-209

75. Petrache HI, Dodd SW, Brown MF (2000) Biophys J 79:3172-3192

76. Nagle JF, Tristram-Nagle S (2000) Biochim Biophys Acta Rev Biomembr 1469:159-195

77. Janiak MJ, Small DM, Shipley GG (1979) J Lipid Res 20:183-199

78. Gunasekara L, Schurch S, Schoel WM (2005) Biochimi Biophys Acta Mol Cell Biol Lipids 1737:27-35

79. Leonenko Z, Finot E, Amrein M (2007) Ultramicroscopy 107:948-953

80. Leonenko Z, Rodenstein M, Dohner J, Eng LM, Amrein M (2006) Langmuir 22:1013510139

81. Leonenko Z, Gill S, Baoukina S, Monticelli L, Doehner J, Gunasekara L, Felderer F, Rodenstein M, Eng LM, Amrein M (2007) Biophys J 93:674-683

82. Chiu SW, Jakobsson E, Scott HL (2001) J Chem Phys 114:5435-5443

83. Smondyrev AM, Berkowitz ML (1999) J Chem Phys 111:9864-9870

84. Smondyrev AM, Berkowitz ML (1999) J Chem Phys 110:3981-3985

85. Atkinson RW, Anderson HR, Strachan DP, Bland JM, Bremner SA, de Leon AP (1999) Eur Respir J 13:257-265

86. Oberdorster G, Sharp Z, Atudorei V, Elder A, Gelein R, Lunts A, Kreyling W, Cox C (2002) J Toxicol Environ Health 65:1531-1543

87. Geiser M, Rothen-Rutishauser B, Kapp N, Schurch S, Kreyling W, Schulz H, Semmler M, Hof VI, Heyder J, Gehr P (2005) Environ Health Perspect 113:1555-1560

88. Nemmar A, Hoet PHM, Vanquickenborne B, Dinsdale D, Thomeer M, Hoylaerts MF, Vanbilloen H, Mortelmans L, Nemery B (2002) Circulation 105:411-414

89. Takenaka S, Karg E, Roth C, Schulz H, Ziesenis A, Heinzmann U, Schramel P, Heyder J (2001) Environ Health Perspect 109:547-551

90. Chen H, Langer R, Edwards DA (1997) J Colloid Interface Sci 190:118-133

91. Bresme F, Quirke N (1999) J Chem Phys 110:3536-3547

92. Shanahan MER (1990) J Phys D Appl Phys 23:321-327

93. Le C Grimellec, Lesniewska E, Giocondi MC et al (1998) Biophys J 75:695-703

94. Costa KD (2003) Dis Markers 19:139-154

95. Radmacher M (2002) Methods Cell Biol 68:67-90

96. Sneddon JN (1965) Int J Eng Sci 3:47

97. Mc MElfresh, Baesu E, Balhorn R, Belak J, Allen MJ, Rudd RE (2002) Proc Natl Acad Sci USA 99:6493-6497

98. Stark RW, Drobek T, Weth M, Frickef J, Heckel WM (1998) Ultramicroscopy 75:161

99. Radmacher M, Fritz M, Kacher CM, Cleveland JP, Hansma PK (1996) Biophys J 70:556567

100. Mathur AB, Collinsworth AM, Reichert WM, Kraus WE, Truskey GA (2001) J Biomech 34:1545-1553

101. Bachofen H, Schurch S, Possmayer F (1994) J Appl Physiol 76:1983-1992

102. Gehr P, Geiser M, Im V Hof, Schurch S, Waber U, Baumann M (1993) Microsc Res Tech 26:423-436

103. Biggs S, Spinks G (1998) J Adhes Sci Technol 12:461-478

104. Reitsma M, Craig VSJ, Biggs S (2000) J Adhes 74:125-142

105. Heim LO, Blum J, Preuss M, Butt H-J (1999) Phys Rev Lett 83:3328-3331 
106. Geiser M, Rothen-Rutishauser B, Kapp N, Schurch S, Kreyling W, Schulz H, Semmler M, Hof VI, Heyder J, Gehr P (2005) Environ Health Perspect 113:1555-1560

107. Rothen-Rutishauser BM, Schurch S, Haenni B, Kapp N, Gehr P (2006) Environ Sci Technol 40:4353-4359

108. Blank F, Rothen-Rutishauser BM, Schurch S, Gehr P (2006) J Aerosol Med 19 (in press) 